\title{
MAPPINGS OF FINITE DISTORTION: FORMATION OF CUSPS III
}

\author{
PEKKA KOSKELA AND JUHANI TAKKINEN
}

\begin{abstract}
We give sharp integrability conditions on the distortion of a planar homeomorphism that maps a standard cusp onto the unit disk.
\end{abstract}

\section{INTRODUCTION}

In this paper, we consider homeomorphisms $f: \mathbb{R}^{2} \rightarrow \mathbb{R}^{2}$ so that $f \in W_{\text {loc }}^{1,1}\left(\mathbb{R}^{2} ; \mathbb{R}^{2}\right), J_{f}(x) \geq 0$ almost everywhere and $D f(x)=0$ almost everywhere in the zero set of the Jacobian determinant $J_{f}$ of $f$. It then immediately follows that

$$
|D f(x)|^{2} \leq K(x) J_{f}(x)
$$

almost everywhere, where $1 \leq K(x)<\infty$. The optimal such a function $K_{f}$ is given by setting $K_{f}(x)=1$ when $J_{f}(x)=0$ or when $D f(x)$ does not exist and by letting $K_{f}(x)=|D f(x)|^{2} / J_{f}(x)$ otherwise. We then say that $f$ is a homeomorphism of finite distortion and call the above optimal function $K_{f}$ the distortion function of $f$. Since the Jacobian determinant of any homeomorphism $f \in W_{\text {loc }}^{1,1}\left(\mathbb{R}^{2} ; \mathbb{R}^{2}\right)$ is locally integrable (cf. $[6,8]$ ), we deduce that the class of homeomorphisms of bounded distortion coincides with the class of quasiconformal mappings. For the basic properties of these mappings see $[1,2,5,6,8]$. The purpose of this paper is to continue our efforts $[7,9]$ to understand the geometry of the image of the unit disk under a homeomorphism with a nicely integrable distortion function.

Recall that a Jordan domain $\Omega$ is a quasidisk if $\Omega=f(B(0,1))$ for some quasiconformal mapping $f: \mathbb{R}^{2} \rightarrow \mathbb{R}^{2}$. Quasidisks have a geometric characterization in terms of the so-called three point condition that fails for example if the boundary of $\Omega$ contains a cusp. This is in particular the case for our model domains

$$
\Omega_{s}=\left\{\left(x_{1}, x_{2}\right) \in \mathbb{R}^{2}: 0<x_{1}<1,\left|x_{2}\right|<x_{1}^{1+s}\right\} \cup B\left(x_{s}, r_{s}\right),
$$

where $s>0, x_{s}=(s+2,0)$ and $r_{s}=\sqrt{(s+1)^{2}+1}$. Based on our previous work $[7,9]$ we know essentially sharp (exponential integrability) conditions on the distortion function under which $\Omega_{s}$ can arise as the

2000 Mathematics Subject Classification. 30C62,30C65.

Key words and phrases. cusp, homeomorphism, mapping of finite distortion. 
image of the unit disk $B:=B(0,1)$ under a homeomorphism of finite distortion.

Notice that the inverse of a quasiconformal mapping is also quasiconformal. Thus $\Omega_{s}$ cannot be the image of $B$ under a homeomorphism whose inverse is quasiconformal. By a recent result from [4], the inverse of a homeomorphism of finite distortion is also of finite distortion. However, even when $\exp \left(\lambda K_{f}\right)$ is locally integrable, one only has that $K_{f^{-1}}^{c \lambda}$ is locally integrable. By requiring yet higher integrability from $K_{f}$ one could guarantee that $K_{f^{-1}}$ be exponentially integrable and thus apply the known results to rule out $\Omega_{s}$ as the image under a mapping of finite distortion under a non-trivial bound on the distortion of the inverse mapping. Our first result gives a much better conclusion.

Theorem 1. Let $f: \mathbb{R}^{2} \rightarrow \mathbb{R}^{2}$ be a homeomorphism of finite distortion such that $K_{f^{-1}} \in L_{\text {loc }}^{p}\left(\mathbb{R}^{2}\right)$ for some $1 \leq p<\infty$. If $f(B)=\Omega_{s}$ for some $s>0$, then necessarily $s \leq 4 /(p-1)$. If, in addition, $f$ is assumed to be quasiconformal on $B$, then $s \leq 2 / p$.

Our second result shows that the second conclusion in Theorem 1 is optimal and that also the first conclusion is rather optimal.

Theorem 2. For $s>0$ given, there exists a homeomorphism of finite distortion, $f: \mathbb{R}^{2} \rightarrow \mathbb{R}^{2}$, with $f(B)=\Omega_{s}$, such that $K_{f^{-1}} \in L_{\text {loc }}^{p}\left(\mathbb{R}^{2}\right)$ for all $p<2 / s+1$. If one only requires that $K_{f^{-1}} \in L_{\mathrm{loc}}^{p}\left(\mathbb{R}^{2}\right)$ for all $p<2 / s$, then $f$ can be made quasiconformal on $B$.

For the proof of Theorem 1 we establish the following modulus of continuity estimate that we expect to be of independent interest. The optimality of the exponent $p / 2$ below can be seen by considering the radial mapping $f(x)=x|x|^{-1} \log ^{-q}((1+|x|) /|x|)$.

Theorem 3. Let $f: \mathbb{R}^{2} \rightarrow \mathbb{R}^{2}$ a homeomorphism of finite distortion. If $K_{f^{-1}} \in L_{\mathrm{loc}}^{p}\left(\mathbb{R}^{2}\right)$ for some $1 \leq p<\infty$, then for all $|x-y|<1 / 2$

$$
|f(x)-f(y)| \leq \frac{C\left(p,\left\|K_{f^{-1}}\right\|_{L^{p}(G)}\right)}{\log ^{\frac{p}{2}}(1 /|x-y|)},
$$

where $G=f(B(x, 1))$.

We give the necessary definitions in Section 2 below and prove the above theorems in Section 3.

\section{Definitions AND PRELiminaries}

We denote by $B(x, r)$ the open disk of radius $r>0$ centered at $x \in \mathbb{R}^{2}$ and write $B:=B(0,1)$ for the unit disk. The boundary of a set $A$ is denoted by $\partial A$ and the closure by $\bar{A}$.

Let $\Omega \subset \mathbb{R}^{2}$ be a domain, i.e. a connected and open subset of $\mathbb{R}^{2}$. We say that a homeomorphism $f: \Omega \rightarrow f(\Omega) \subset \mathbb{R}^{2}$ has finite distortion if the following conditions are satisfied: 
1. $f \in W_{\text {loc }}^{1,1}\left(\Omega ; \mathbb{R}^{2}\right)$

2. $|D f(x)|^{2} \leq K(x) J_{f}(x)$ a.e. $x \in \Omega$

for some measurable function $K(x) \geq 1$ which is finite almost everywhere. The optimal such a function $K_{f}(x)$ is referred to as the distortion (function) of $f$ and the phrase exponentially integrable distortion means that $\exp \left(\lambda K_{f}\right) \in L_{\text {loc }}^{1}(\Omega)$ for some $\lambda>0$.

Above $D f(x)$ denotes the differential matrix of $f$ at the point $x$ (which for $f \in W_{\text {loc }}^{1,1}\left(\Omega ; \mathbb{R}^{2}\right)$ exists a.e.) and $J_{f}(x):=\operatorname{det} D f(x)$ is the Jacobian. The norm of $D f(x)$ is defined as

$$
|D f(x)|:=\max \left\{|D f(x) e|: e \in \mathbb{R}^{2},|e|=1\right\} .
$$

Let $E$ be a compact set in a domain $G \subset \mathbb{R}^{2}$. The $p$-capacity of the pair $(G, E)$ is defined to be

$$
\begin{array}{r}
\operatorname{cap}_{p}(G, E)=\inf \left\{\int_{G}|\nabla u(x)|^{p} d x: u \in C(G) \cap W_{0}^{1,1}(G),\right. \\
\text { such that } u \geq 1 \text { on } E\} .
\end{array}
$$

For $s>0$ our model cusp domains $\Omega_{s} \subset \mathbb{R}^{2}$ are defined as in (1). The parameter $s$ determines the degree of the cusp, and omitting the origin (tip of the cusp) the boundary of $\Omega_{s}$ is smooth. As already noted, $\Omega_{s}$ is not a quasidisk, because the three point condition fails (only) at the tip of the cusp.

Next lemma provides a capacity -type estimate which is to be used in the proof of Theorem 3 .

Lemma 1. Let $G \subset \mathbb{R}^{2}$ be a bounded domain and $E \subset G$ a continuum. Suppose that $u \in W_{0}^{1,1}(G)$ is continuous and $u \geq 1$ on $E$. Then

$$
\int_{G}|\nabla u|^{p} \geq C(p)(\operatorname{diam} E)^{2-p}
$$

for all $1 \leq p<2$.

Proof. First we extend $u$ as zero to $\mathbb{R}^{2} \backslash G$ and denote also this extension by $u$. Pick $x_{0} \in E$ such that $S\left(x_{0}, r\right) \cap E \neq \emptyset$ for all $d / 2 \leq r \leq$ $d:=\operatorname{diam} E$. Suppose first that $u(x) \geq 1 / 2$ on $S\left(x_{0}, r_{0}\right)$ for some $d / 2 \leq r_{0} \leq d$ and pick $R \geq r_{0}$ such that $G \subset B\left(x_{0}, R\right)$. Notice, that

$$
\tilde{u}(x)= \begin{cases}2 u(x) & \text { when }\left|x-x_{0}\right| \geq r_{0} \\ \max \{1,2 u(x)\} & \text { when }\left|x-x_{0}\right|<r_{0}\end{cases}
$$

is a suitable test function for $\operatorname{cap}_{p}\left(B\left(x_{0}, R\right), \bar{B}\left(x_{0}, r_{0}\right)\right)$ and $|\nabla \tilde{u}| / 2 \leq$ $|\nabla u|$ almost everywhere. As

$$
\operatorname{cap}_{1}\left(B\left(x_{0}, R\right), \bar{B}\left(x_{0}, r_{0}\right)\right)=2 \pi r_{0}
$$

and

$$
\operatorname{cap}_{p}\left(B\left(x_{0}, R\right), \bar{B}\left(x_{0}, r_{0}\right)\right)=C_{0}(p)\left(r_{0}^{\frac{p-2}{p-1}}-R^{\frac{p-2}{p-1}}\right)^{1-p} \geq \tilde{C}_{0}(p) r_{0}^{2-p}
$$


for $1<p<2$ (cf. [6]), we readily obtain the desired estimate

$$
\int_{G}|\nabla u|^{p} \geq 2^{-p} \operatorname{cap}_{p}\left(B\left(x_{0}, R\right), \bar{B}\left(x_{0}, r_{0}\right)\right) \geq C(p) r_{0}^{2-p} \geq \tilde{C}(p) d^{2-p} .
$$

We may thus assume that, for each $d / 2 \leq r \leq d$, there is $y \in S\left(x_{0}, r\right)$ with $u(y) \leq 1 / 2$. Then, for almost every $\bar{d} / 2 \leq r \leq d$

$$
1 / 2 \leq \int_{S\left(x_{0}, r\right)}|\nabla u| \leq(2 \pi r)^{\frac{p-1}{p}}\left(\int_{S\left(x_{0}, r\right)}|\nabla u|^{p}\right)^{\frac{1}{p}}
$$

and so by Fubini

$$
\int_{G}|\nabla u|^{p} \geq \int_{d / 2}^{d} \int_{S\left(x_{0}, r\right)}|\nabla u(x)|^{p} d \omega d r \geq \int_{d / 2}^{d} \frac{d r}{2^{p}(2 \pi r)^{p-1}}=C(p) d^{2-p},
$$

which proves the claim.

\section{Proofs of the Theorems}

Proof of Theorem 1. To simplify calculations, we may assume that $f((1,0))=(0,0)$. Let $0<h<1 / 2$ and define

$$
\begin{aligned}
& E^{\prime}:=\left\{\left(x_{1}, x_{2}\right) \in \partial \Omega_{s}: h \leq x_{1} \leq 1 / 2, x_{2}>0\right\} \\
& F^{\prime}:=\left\{\left(x_{1}, x_{2}\right) \in \partial \Omega_{s}: h \leq x_{1} \leq 1 / 2, x_{2}<0\right\},
\end{aligned}
$$

so that $\operatorname{dist}\left(E^{\prime}, F^{\prime}\right)=2 h^{1+s}$. Set $E:=f^{-1}\left(E^{\prime}\right)$ and $F:=f^{-1}\left(F^{\prime}\right)$, which will be separate continua and subsets of $\partial B$ because $f^{-1}$ is a homeomorphism (see Figure 1). Furthermore, as $(0,0) \notin E^{\prime} \cup F^{\prime}$, it will be that $(1,0) \notin E \cup F$. Set $\tilde{h}:=\min \{\operatorname{dist}((1,0), E)$, $\operatorname{dist}((1,0), F)$ and $C_{f}:=\frac{1}{2} \operatorname{dist}\left(f^{-1}\left(\left(1 / 2,1 / 2^{1+s}\right)\right), f^{-1}\left(\left(1 / 2,-1 / 2^{1+s}\right)\right)\right)$. Now, $C_{f}>0$ and by taking $h$ small enough in the beginning, we may assume that $0<\tilde{h}<1 / 2$. Notice also that $C_{f}$ does not depend on $h$ or $\tilde{h}$.

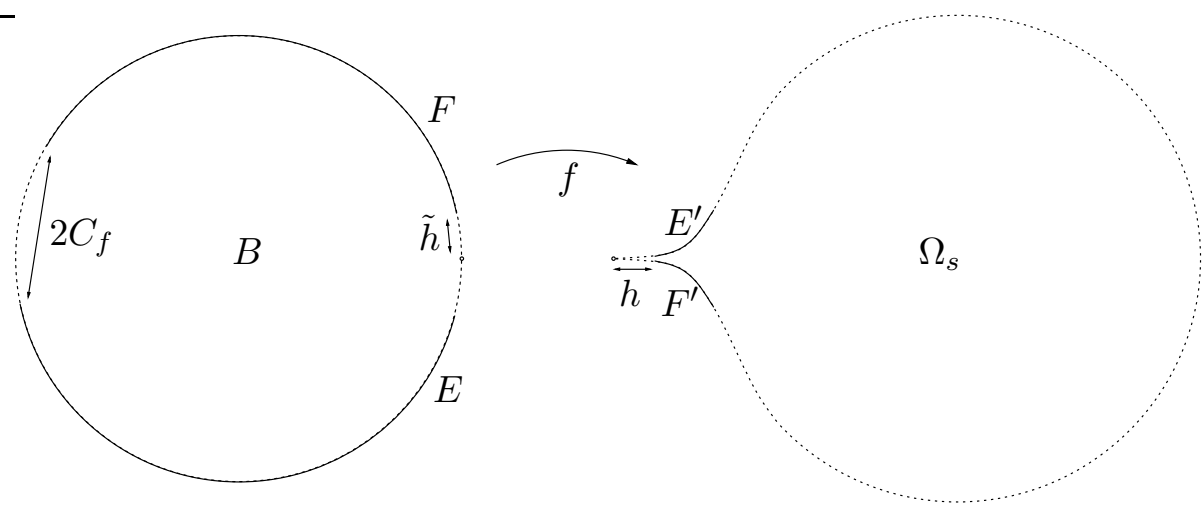

FiguRE 1 . The setting in the proof of Theorem 1

Let

$$
\rho(x)= \begin{cases}1 /\left(C_{f}|x-(1,0)|\right) & \text { for }|x-(1,0)|>\tilde{h} \\ 1 /\left(C_{f} \tilde{h}\right) & \text { for }|x-(1,0)| \leq \tilde{h}\end{cases}
$$


and define $v(x)=\inf \int_{\gamma_{x}} \rho d s$, where the infimum is taken over all rectifiable paths $\gamma_{x}$ in $\bar{B}$ joining $x \in \bar{B}$ to $E$. One easily observes that $v(x)=0$ for all $x \in E$ and $v(x) \geq 1$ for all $x \in F$. Moreover, as $\nabla v=\rho$ in $B$, we easily obtain an upper estimate for $\int_{B}|\nabla v|^{2}$ by computing

$$
\int_{B}|\nabla v|^{2} \leq C_{f}^{-2}\left(\pi+\int_{B \backslash B((1,0), \tilde{h})} \frac{1}{|x-(1,0)|^{2}}\right) \leq \tilde{C}_{f} \log (1 / \tilde{h}) .
$$

Let $q=2 p /(p+1)$ and set $u=v \circ f^{-1}$, so that now $u \in W^{1,1}\left(\Omega_{s}\right)$ with $u=0$ on $E^{\prime}$ and $u \geq 1$ on $F^{\prime}$. Thus for almost every $t \in[h, 1 / 2]$ we have

$$
1 \leq \int_{-t^{1+s}}^{t^{1+s}}|\nabla u(t, y)| d y \leq\left(\int_{-t^{1+s}}^{t^{1+s}}|\nabla u(t, y)|^{q} d y\right)^{\frac{1}{q}}\left(2 t^{1+s}\right)^{\frac{q-1}{q}} .
$$

In the case of $(s+1)(q-1) \leq 1$ we readily obtain $s \leq 2 /(p-1)$ and are done. Thus we may assume that $(s+1)(q-1)>1$. In this case $(6)$ implies via Fubini that

$$
\int_{\Omega_{s}}|\nabla u|^{q} \geq \int_{h}^{1} \frac{2^{1-q}}{t^{(1+s)(q-1)}} d t \geq \frac{C_{0}}{h^{(s+1)(q-1)-1}} .
$$

On the other hand, by applying the distortion inequality and the Hölder's inequality, we obtain the upper estimate

$$
\begin{aligned}
\int_{\Omega_{s}}|\nabla u|^{q} & \leq \int_{\Omega_{s}}\left|\nabla v\left(f^{-1}\right)\right|^{q}\left|D f^{-1}\right|^{q} \\
& \leq \int_{\Omega_{s}}\left|\nabla v\left(f^{-1}\right)\right|^{q} J_{f^{-1}}^{\frac{q}{2}} K_{f^{-1}}^{\frac{q}{2}} \\
& \leq\left(\int_{\Omega_{s}}\left|\nabla v\left(f^{-1}\right)\right|^{2} J_{f^{-1}}\right)^{\frac{q}{2}}\left(\int_{\Omega_{s}}\left[K_{f^{-1}}\right]^{\frac{q}{2-q}}\right)^{\frac{2-q}{2}} \\
& \leq C\left(f,\left\|K_{f^{-1}}\right\|_{L^{p}\left(\Omega_{s}\right)}\right) \log ^{\frac{q}{2}}(1 / \tilde{h}) .
\end{aligned}
$$

Here the last inequality follows from a change of variable (cf. [6], Theorem 6.3.2), the estimate (5) and the fact that $q /(2-q)=p$.

By applying the modulus of continuity estimate (2) to (7) and combining the result with (8), we obtain the estimate

$$
C_{1} \log ^{\frac{p}{2}((s+1)(q-1)-1)}(1 / \tilde{h}) \leq C_{2} \log ^{\frac{q}{2}}(1 / \tilde{h}) .
$$

By substituting $q=2 p /(p+1)$, and observing that this inequality must hold for all small $\tilde{h}$, we readily have

$$
\frac{p}{2}\left((s+1)\left(\frac{2 p}{p+1}-1\right)-1\right) \leq \frac{p}{p+1},
$$

which, when solving for $s$, implies that $s \leq 4 /(p-1)$.

If $f$ is assumed to be quasiconformal on $B$, we can take $q=2$, and have the lower bound

$$
\int_{\Omega_{s}}|\nabla u|^{2} \geq \frac{C_{0}}{h^{s}}
$$


and the upper bound

$$
\begin{aligned}
\int_{\Omega_{s}}|\nabla u|^{2} & \leq \int_{\Omega_{s}}\left|\nabla v\left(f^{-1}\right)\right|^{2}\left|D f^{-1}\right|^{2} \leq \int_{\Omega_{s}}\left|\nabla v\left(f^{-1}\right)\right|^{2} J_{f^{-1}} K_{f^{-1}} \\
& \leq\left\|K_{f^{-1}}\right\|_{L^{\infty}\left(\Omega_{s}\right)} \int_{B}|\nabla v|^{2} \leq C\left(f,\left\|K_{f^{-1}}\right\|_{L^{\infty}\left(\Omega_{s}\right)}\right) \log (1 / \tilde{h}) .
\end{aligned}
$$

By applying the modulus of continuity to the lower bound and combining the result with the upper bound, one arrives via similar reasoning as in the first part of the proof to the inequality $s(p / 2) \leq 1$, which proves the last part of the claim.

Proof of Theorem 2. First we consider the case when $f$ is not required to be quasiconformal on $B$. Set $Q=\left\{(x, y) \in \mathbb{R}^{2}:|x| \leq 1\right.$ and $|y| \leq$ $1\}, Q^{+}=Q \cap\left\{(x, y) \in \mathbb{R}^{2}: x \geq 0\right\}$ and $Q^{-}=Q \cap\left\{(x, y) \in \mathbb{R}^{2}: x<0\right\}$, and define

$$
h(x, y)= \begin{cases}\left(x, y x^{-s}\right) & \text { if }(x, y) \in Q^{+} \cap \Omega_{s}, \\ \left(x, y^{\frac{1}{1+s}}\right) & \text { if }(x, y) \in Q^{+} \backslash \Omega_{s} .\end{cases}
$$

This gives a homeomorphic self map on $Q^{+}$such that the set $\{(x, y) \in$ $\left.Q^{+}:|y|=x^{1+s}\right\}=Q^{+} \cap \partial \Omega_{s}$ maps to the set $\left\{(x, y) \in Q^{+}:|y|=x\right\}$, and the mapping is identity on $\partial Q^{+} \backslash\left\{(x, y) \in \mathbb{R}^{2}: x=0,-1<y<1\right\}$. Next we define a mapping $H: \mathbb{R}^{2} \rightarrow \mathbb{R}^{2}$ by setting

$$
H(x, y)= \begin{cases}h(x, y) & \text { if }(x, y) \in Q^{+} \\ (T \circ h \circ T)(x, y) & \text { if }(x, y) \in Q^{-} \\ (x, y) & \text { if }(x, y) \in \mathbb{R}^{2} \backslash Q\end{cases}
$$

where $T: \mathbb{R}^{2} \rightarrow \mathbb{R}^{2}$ is the reflection with respect to the $y$-axis, i.e. $(x, y) \mapsto(-x, y)$. It is easily seen that $H$ is homeomorphic and that it "opens" the only zero angle of $\partial \Omega_{s}$ that resides at the origin. Because $H\left(\Omega_{s}\right)$ will clearly be a quasidisk, there exists a quasiconformal mapping $b: \mathbb{R}^{2} \rightarrow \mathbb{R}^{2}$ with $b\left(H\left(\Omega_{s}\right)\right)=B$.

Next we will show that the composition $b \circ H: \mathbb{R}^{2} \rightarrow \mathbb{R}^{2}$, that is clearly homeomorphic, is also a mapping of finite distortion. The desired mapping $f$ will then be the inverse of $b \circ H$ as Theorem 3.3. in [4] states that the inverse of a homeomorphism of finite distortion is also of finite distortion. As we are interested on the local $L^{p_{-}}$ integrability of $K_{b \circ H}$, we only need to consider $K_{H}$ as we can take $K_{b \circ H}(x)=K_{b}(H(x)) K_{H}(x)$, where $K_{b}$ is bounded.

On the set $\mathbb{R}^{2} \backslash Q$ the distortion of $H$ is evidently bounded, and because of the symmetry, we only need to consider the set $Q^{+}$. As the differential matrices of $H$ in $Q^{+} \cap \Omega_{s}$ and $Q^{+} \backslash \Omega_{s}$ are

$$
\left[\begin{array}{cc}
1 & 0 \\
-s y x^{-s-1} & x^{-s}
\end{array}\right] \quad \text { and } \quad\left[\begin{array}{cc}
1 & 0 \\
0 & \frac{1}{1+s} y^{-s /(1+s)}
\end{array}\right] \text {, }
$$


respectively, we observe that $K_{H}(x, y) \asymp 1 / x^{s}$ when $(x, y) \in Q^{+} \cap \Omega_{s}$ and $K_{H}(x, y) \asymp 1 / y^{-s /(1+s)}$ when $(x, y) \in Q^{+} \backslash \Omega_{s}$. Next, by computing

$$
\int_{Q^{+} \cap \Omega_{s}} K_{H}^{p} \asymp \int_{0}^{1} \int_{-x^{1+s}}^{x^{1+s}} x^{-p s} d y d x=\int_{0}^{1} 2 x^{1+s-p s} d x
$$

and

$$
\int_{Q^{+} \backslash \Omega_{s}} K_{H}^{p} \asymp \int_{0}^{1} \int_{x^{1+s}}^{1} y^{-p s /(1+s)} d y d x \asymp \int_{0}^{1} x^{1+s-p s} d x
$$

we see that $K_{H} \in L_{\text {loc }}^{p}\left(\mathbb{R}^{2}\right)$ if $1+s-p s>-1$, i.e. $p<2 / s+1$.

Next we consider the situation when $f$ is quasiconformal on $B$. Here we refer to the mapping constructed in [7] in the proof of Theorem 1. For a given $s>0$, a homeomorphism $f: \mathbb{R}^{2} \rightarrow \mathbb{R}^{2}$ of finite distortion is constructed which maps $B$ to $\Omega_{s}$ and is quasiconformal on $B$. The point $a:=(-1,0) \in \partial B$ maps to the tip of the cusp $(0,0) \in \partial \Omega_{s}$. Also, for some $0<r_{0}<1$ the distortion is bounded outside $B\left(a, r_{0}\right)$ and in $B\left(a, r_{0}\right)$ we have that

$$
K_{f}(x) \asymp \log (2 /|x-a|) .
$$

Moreover, this specific mapping satisfies

$$
|f(x)| \leq C \log ^{-1 / s}(2 /|x-a|),
$$

for all $0<|x-a|<1$ so that

$$
\left|f^{-1}(y)-a\right| \geq 2 \exp ^{-1}\left(\tilde{C}|y|^{-s}\right)
$$

when $|y|$ is small.

As $f$ is a homeomorphism, we may choose $0<r^{\prime}<1$ such that $f^{-1}\left(B\left(0, r^{\prime}\right)\right) \subset B\left(a, r_{0}\right)$. Now, by applying (9) and (10) and using the fact that $K_{f^{-1}}(y)=K_{f}\left(f^{-1}(y)\right)$ (cf. [4], proof of Theorem 1.6, and [3], Corollary 2.2) we may estimate $\int_{B\left(0, r^{\prime}\right)} K_{f^{-1}}^{p}$ by computing

$$
\begin{aligned}
\int_{B\left(0, r^{\prime}\right)} K_{f^{-1}}^{p}(y) d y & =\int_{B\left(0, r^{\prime}\right)} K_{f}^{p}\left(f^{-1}(y)\right) d y \\
& \leq C_{1} \int_{B\left(0, r^{\prime}\right)} \log ^{p}\left(\frac{2}{\left|f^{-1}(y)-a\right|}\right) d y \\
& \leq C_{2} \int_{B\left(0, r^{\prime}\right)} \frac{d y}{|y|^{p s}}
\end{aligned}
$$

As the last integral is finite when $p s<2$, i.e. $p<2 / s$, the claim is proven.

Proof of Theorem 3. Set $q:=2 p /(1+p)$ so that $1 \leq q<2$. Let $x$, $y \in \mathbb{R}^{2}$ be such that $y \in B(x, 1 / 2)$ and set $F \subset B(x, 1):=B^{\prime}$ to be the line segment between these points. Denote $G:=f\left(B^{\prime}\right)$ and $E:=f(F)$. Now, Lemma 1 readily implies that

$$
\operatorname{cap}_{q}(G, E) \geq C(q)(\operatorname{diam} E)^{2-q} \geq C(q)|f(x)-f(y)|^{2-q} .
$$


Let $u$ be a Lipschitz continuous test function for $\operatorname{cap}_{2}\left(B^{\prime}, F\right)$ and define $v=u \circ f^{-1}$, which will give a test function for $\operatorname{cap}_{q}(G, E)$. By applying the distortion inequality, Hölders inequality and a change of variable, we arrive at the estimate

$$
\begin{aligned}
\operatorname{cap}_{q}(G, E) & \leq \int_{G}|\nabla v|^{q} \leq \int_{G}\left|\nabla u\left(f^{-1}\right)\right|^{q}\left|D f^{-1}\right|^{q} \\
& \leq \int_{G}\left[\left|\nabla u\left(f^{-1}\right)\right|^{2} J_{f^{-1}}\right]^{q / 2} K_{f^{-1}}^{q / 2} \\
& \leq\left(\int_{B^{\prime}}|\nabla u|^{2}\right)^{q / 2}\left(\int_{G} K_{f^{-1}}^{q /(2-q)}\right)^{(2-q) / 2} .
\end{aligned}
$$

By approximating an arbitrary test function $\tilde{u}$ for $\operatorname{cap}_{2}\left(B^{\prime}, F\right)$ by Lipschitz continuous test functions, we obtain

$$
\operatorname{cap}_{q}(G, E) \leq\left[\operatorname{cap}_{2}\left(B^{\prime}, F\right) \cdot\left\|K_{f^{-1}}\right\|_{L^{q /(2-q)}(G)}\right]^{q / 2} .
$$

By combining (11) and (12), and using the standard capacity estimate $\operatorname{cap}_{2}\left(B^{\prime}, F\right) \leq 2 \pi \log ^{-1}\left(\operatorname{diam} B^{\prime} / \operatorname{diam} F\right)$ for the pair $\left(B^{\prime}, F\right)$, we conclude that

$$
C(q)|f(x)-f(y)|^{2-q} \leq\left(2 \pi \log ^{-1}(1 /|x-y|)\left\|K_{f^{-1}}\right\|_{L^{q /(2-q)}(G)}\right)^{q / 2},
$$

which proves $(2)$ as $p=q /(2-q)$.

\section{REFERENCES}

1. K. Astala, T. Iwaniec, P. Koskela, and G. Martin, Mappings of BMO-bounded distortion, Math. Ann. 317 (2000), no. 4, 703-726.

2. K. Astala, T. Iwaniec, G. J. Martin, and J. Onninen, Extremal mappings of finite distortion, Proc. London Math. Soc. (3) 91 (2005), no. 3, 655-702.

3. L. Greco, C. Sbordone, and C. Trombetti, A note on planar homeomorphisms, Preprint.

4. S. Hencl and P. Koskela, Regularity of the inverse of a planar Sobolev homeomorphism, Arch. Ration. Mech. Anal. 180 (2006), no. 1, 75-95.

5. T. Iwaniec, P. Koskela, and G. Martin, Mappings of BMO-distortion and Beltrami-type operators. Dedicated to the memory of Tom Wolff, J. Anal. Math. 88 (2002), 337-381.

6. T. Iwaniec and G. Martin, Geometric function theory and non-linear analysis, Oxford Mathematical Monographs. The Clarendon Press, Oxford University Press, New York, 2001.

7. P. Koskela and J. Takkinen, Mappings of finite distortion: formation of cusps, Publ. Mat. 51 (2007), no. 1, 223-242.

8. O. Lehto and K.I. Virtanen, Quasiconformal mappings in the plane, second ed., Springer-Verlag, New York, 1973.

9. J. Takkinen, Mappings of finite distortion: formation of cusps II, Conform. Geom. Dyn., to appear. 
(Pekka Koskela) Department of Mathematics and Statistics, UniverSity of JyvÄskylä, P.O. BoX 35, FI-40014 University OF JyV̈̈SKYLÄ, FinLAND

E-mail address: pkoskela@maths.jyu.fi

(Juhani Takkinen) Department of Mathematics and Statistics, UniverSity of JyvÄskyl ̈̈, P.O. Box 35, FI-40014 University of JyväSKYLÄ, FinLAND

E-mail address: juhani@maths.jyu.fi 